\title{
Mild versus Severe Liver Injury in SARS-CoV-2 Infection
}

\author{
Olympia E. Anastasiou ${ }^{a} \quad J^{\prime}$ Johannes Korth ${ }^{\mathrm{b}}$ Frank Herbstreit ${ }^{\mathrm{c}} \quad$ Oliver Witzke $^{\mathrm{d}}$ \\ Christian M. Lange ${ }^{\mathrm{e}}$ \\ anstitute for Virology, University Hospital Essen, University of Duisburg-Essen, Essen, Germany; ${ }^{\text {bDepartment }}$ \\ of Nephrology, University Hospital Essen, University of Duisburg-Essen, Essen, Germany; 'Department of \\ Anesthesiology and Intensive Care, Essen University Hospital, Essen, Germany; ${ }^{d}$ Department of Infectious Diseases, \\ West German Centre of Infectious Diseases, Universitätsmedizin Essen, University Duisburg-Essen, Essen, Germany; \\ eDepartment of Gastroenterology and Hepatology, University Hospital Essen, Essen, Germany
}

\section{Keywords}

Coronavirus disease 2019. Severe acute respiratory syndrome coronavirus- 2 . Acute liver failure $\cdot$ Viral kinetics

\begin{abstract}
Background: Abnormal liver function has been reported in patients with COVID-19 infection. The aim of our study was to report on the prevalence of liver injury in our cohort, to evaluate the association of mild versus severe liver injury with mortality in COVID-19 patients and to scrutinize the temporal pattern of viral detection and liver injury. Methods: We present data from a German cohort of 147 SARS-CoV-2 infected patients. The patients were divided into 3 groups according to their liver status during treatment. The first group included patients without elevated alanine aminotransferase or bilirubin, the third group patients meeting the biochemical criteria of acute liver failure (ALF), and the second group all other patients. Results: Liver injury was detected in $75(50.7 \%)$ and 93 (63\%) patients by admission and during treatment, respectively. ALF was associated with the male sex, younger age, and higher BMI. Mortality was associated with the presence of ALF (OR $=9.423,95 \% \mathrm{Cl}: 2.410$ 36.858 ) in contrast to milder liver injury (OR $1.101,95 \% \mathrm{Cl}$ :
\end{abstract}

$0.435-2.791)$. In $30 \%$ of patients with mild liver injury and in $50 \%$ of ALF patients, peak liver injury was observed at a time point when the virus was no longer detectable in the respiratory tract. Conclusion: Mild liver injury was not associated with worse outcome in our cohort, and the pattern of liver injury did not fit well to the theory of SARS-CoV-2 directly causing liver impairment. Instead, severe liver injury in our cohort was associated multiple-organ failure and acute vascular events.

c) 2020 S. Karger AG, Basel

\section{Introduction}

Severe acute respiratory syndrome coronavirus-2 (SARS-CoV-2), the cause of coronavirus disease 2019 (COVID-19), has caused a pandemic with significant repercussions for not only the public health but also the global economy $[1,2]$. Respiratory symptoms are central to the disease; however, nonrespiratory symptoms have been frequently observed as well [3-5]. Abnormal liver function has been reported in COVID-19 patients, and its prevalence ranges from 16.1 to $66.6 \%$ depending on the patient cohort and the definition of abnormality $[6,7]$.

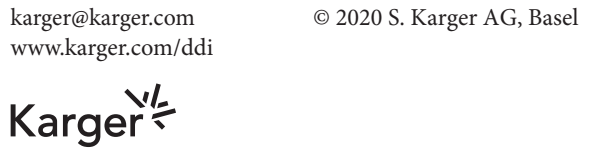

Olympia E. Anastasiou

Institute for Virology, University of Duisburg-Essen Hufelandstraße 55

DE-45147 Essen (Germany)

olympia.anastasiou@uni-due.de 
Two previous studies have reported an association between abnormal liver function at admission and severity of COVID-19 infection $[6,8]$, while another found no such association after adjusting for multiple confounding factors [9]. It remains to date unclear if the virus can cause direct damage to the liver or if the observed liver injury is indirectly caused, possibly immune-mediated or due to drug-induced liver injury or multiorgan failure. The aim of our study was to report on the prevalence of liver injury in our cohort, to evaluate the association of mild versus severe liver injury with mortality in COVID-19 patients and to scrutinize the temporal pattern of viral detection and liver injury.

\section{Patients and Methods}

We report data from a German cohort of 147 SARS-CoV-2 infected patients, diagnosed from Mar 3 to May 14, 2020, with SARSCoV-2 infection with PCR and treated in the Essen University Hospital. Demographical, clinical, and virological data on admission and at peak liver injury were taken from patient charts and are presented in Table 1. Peak liver injury was defined as the time point of the highest MELD score for patients with acute liver failure (ALF) and highest bilirubin or alanine aminotransferase (ALT) (if bilirubin was normal) for all other patients. The patients were divided into 3 groups of liver injury according to their status during treatment. The first group included patients without elevated ALT $(<35 \mathrm{U} / \mathrm{L})$ or bilirubin $(<2 \mathrm{mg} / \mathrm{dL})$, the second group patients with elevated ALT and/or bilirubin but without meeting the biochemical criteria of ALF, and the third group patients meeting the biochemical criteria of ALF, since due to sedation data on hepatic encephalopathy are limited [10]. There was no specific treatment of ALF in all but one patient. One patient received, however, extracorporal liver support therapy using the Prometheus system. ALT was chosen as a marker of liver injury for grouping purposes. ALT is more specific for hepatocellular injury compared to aspartate aminotransferase (AST). ALT is found predominantly in the liver with insignificant concentrations in other organs, while AST is found also in the heart, skeletal muscle, kidneys, brain, and red blood cells. ALT is also more indicative of hepatocellular injury than alkaline phosphatase (ALP) and $\gamma$-glutamyl transpeptidase (GGT), which can be elevated in a variety of nonhepatic conditions, including chronic obstructive pulmonary disease and more importantly for our cohort, renal failure and following cardiovascular events [11]. The upper normal limit for ALT, AST, or GGT was 35 U/L. Comorbidities before and after SARS-CoV-2 infection were documented, focusing especially on acute vascular events after SARS-CoV-2 infection.

Virological Parameters

SARS-CoV-2 RNA was detected using RealStar ${ }^{\circledR}$ SARS-CoV-2 RT-PCR (Altona Diagnostics, Hamburg, Germany) or Abbott RealTime SARS-CoV-2 (Abbott Laboratories, Chicago, IL, USA) according to the manufacturer's instructions. Tested material included material from the respiratory tract (nasopharyngeal swabs, bronchoalveolar lavage, and endotracheal aspirates) and in some cases stool, urine, plasma, and liver biopsies. Viral shedding was measured in days, starting from the first positive time point and ending to the last positive. Patients in whom the interval between the last positive and first negative PCR was $>10$ days were excluded from the viral shedding analysis. Thus, data were available in 57 cases. We also evaluated the timeline of liver injury and viral detection and, more specifically, if the time point of peak liver injury coincided with the presence or absence of the virus in the respiratory tract. Patients were included in this analysis if a PCR for SARS-CoV-2 was performed the same day as the peak liver injury occurred or if the peak liver injury took place between 2 positive or 2 negative PCR results.

\section{Statistical Analysis}

Categorical data were expressed as number (percentage) and continuous data as median (interquartile range). Normality of distribution was tested with the Shapiro-Wilks test. Statistical significance was assessed by Fisher's exact test for categorical data and Mann-Whitney U, ANOVA, or Kruskal-Wallis tests for continuous variables. Univariate and multivariate logistic regression analysis was used to identify factors associated with mortality and liver injury. Parameters with a significant association in the univariate model were included in the multivariate analysis. Two-tailed $p$ values $<0.05$ were considered statistically significant. Statistical analysis was performed using SPSS software (v21, SPSS Inc., Chicago, IL, USA) and the platform "VassarStats."

\section{Results}

\section{Liver Injury Was Common in Patients with SARS-}

CoV-2 Infection and Associated with the Male Sex

Abnormal liver parameters were common in our patient cohort at admission and during treatment, amounting to $123(83.1 \%)$ and $130(87.8 \%)$ patients, respectively. Liver injury (increased ALT and/or bilirubin) was present in $75(50.7 \%)$ patients by admission and in $93(63 \%)$ patients during treatment, while $11(6.8 \%)$ patients developed ALF. ALF patients were younger and had a higher BMI than patients without or with mild liver injury. Acute vascular events were more often diagnosed in the ALF group, and the difference, however, did not reach statistical significance. Liver injury was associated with increased inflammation parameters: patients with mild and severe liver injury had higher leucocyte count and C-reactive protein (CRP) at admission than patients without liver injury (Table 1). All ALF patients presented with or developed sepsis and multiple organ dysfunction syndrome (MODS); additionally 1 patient developed pulmonary embolism, mesenterial ischemia, and liver and spleen infarction, 1 patient spleen infarction, and another pulmonary embolism. 
Table 1. Clinical and virological characteristics of our cohort

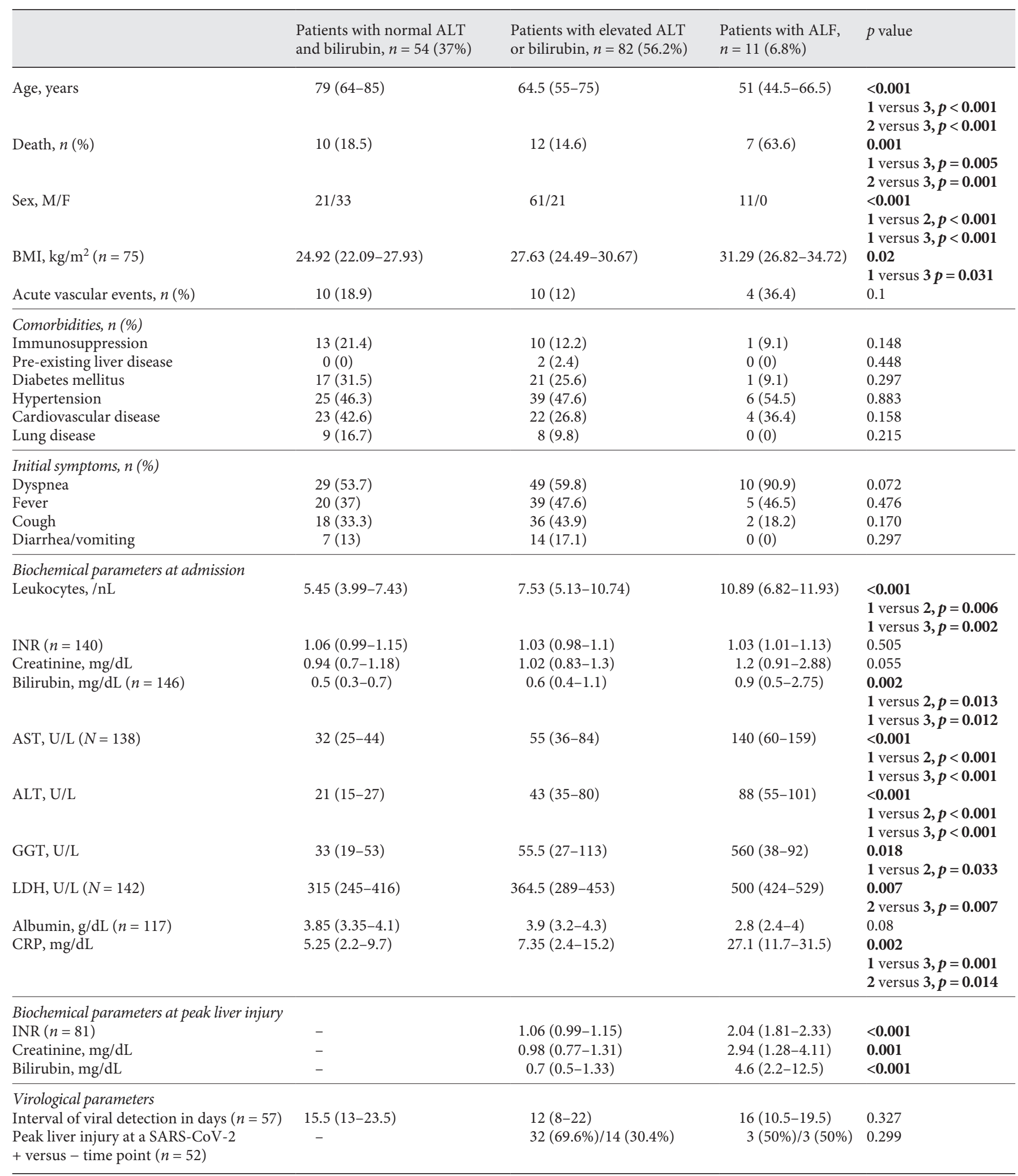

Data are presented as $n$ (\%) or median (interquartile range). ALF, acute liver failure; BMI, body mass index; INR, international normalized ratio; AST, aspartate aminotransferase; ALT, alanine aminotransferase; GGT, $\gamma$-glutamyltransferase; LDH, lactate dehydrogenase; CRP, C-reactive protein. 
Table 2. Parameters associated with mortality on COVID-19 patients

\begin{tabular}{|c|c|c|c|c|}
\hline & \multicolumn{2}{|l|}{ Univariate analysis } & \multicolumn{2}{|l|}{ Multivariate analysis } \\
\hline & OR (95\% CI) & $p$ value & OR $(95 \% \mathrm{CI})$ & $p$ value \\
\hline Age, years & $1.041(1.011-1.072)$ & 0.007 & $1.080(1.037-1.125)$ & $<0.001$ \\
\hline Sex & $0.596(0.244-1.458)$ & 0.257 & - & \\
\hline BMI $\left(\mathrm{kg} / \mathrm{m}^{2}\right)$ & $1.045(0.982-1.112)$ & 0.166 & - & \\
\hline Arterial hypertension & $2.496(1.069-5.828)$ & 0.034 & & ns \\
\hline Diabetes mellitus & $0.672(0.251-1.798)$ & 0.429 & - & \\
\hline Cardiovascular disease & $2.213(0.967-5.069)$ & 0.06 & - & \\
\hline Lung disease & $1.167(0.312-4.363)$ & 0.819 & - & \\
\hline \multirow[t]{4}{*}{ Liver injury during treatment ${ }^{\mathrm{a}}$} & 1 versus 2 & 0.839 & 1 versus 2 & 0.231 \\
\hline & $1.101(0.435-2.791)$ & 0.001 & $1.910(0.663-5.498)$ & $<0.001$ \\
\hline & 1 versus 3 & & 1 versus 3 & \\
\hline & $9.423(2.410-36.858)$ & & $67.437(8.432-539.341)$ & \\
\hline Immunosuppression & $0.533(0.147-1.926)$ & 0.337 & - & \\
\hline Acute vascular events & $2.429(0.921-6.406)$ & 0.073 & - & \\
\hline
\end{tabular}

BMI, body mass index; OR, odds ratio; CI, confidence interval; ALT, alanine aminotransferase; ALF, acute liver failure. ${ }^{\text {a }} 1$, patients with normal ALT and bilirubin; 2, patients with elevated ALT or bilirubin; 3, patients with ALF.

Mild Liver Injury during Treatment Was Not

Associated with Increased Mortality Compared to

Patients without Liver Injury

Several parameters were evaluated in conjunction with their potential association with mortality. Abnormal liver parameters and liver injury on admission were not significantly associated with increased mortality in our cohort ( $p=0.417$ and $p=0.107$, respectively). As shown in Table 2, sex, BMI, presence of diabetes mellitus, lung or cardiovascular comorbidities prior to COVID-19 diagnosis, immunosuppression, and the diagnosis of acute vascular events at and after admission demonstrated no significant association with increased mortality. However, arterial hypertension ( $\mathrm{OR}=2.496,95 \%$ CI: $1.069-$ $5.828, p=0.034)$, age (OR $=1.041,95 \%$ CI: $1.011-1.072$, $p=0.007)$, and the degree of liver injury during treatment (group 1 vs. $2, \mathrm{OR}=1.101,95 \% \mathrm{CI}$ : $0.435-2.791, p=0.839$; group 1 vs. $3, \mathrm{OR}=9.423,95 \%$ CI: $2.410-36.858, p=$ $0.001)$ were significantly associated with mortality in univariate logistic regression analysis. The aforementioned parameters were tested in a multivariate logistic regression model. Age (OR = 1.080, 95\% CI: 1.037-1.125, $p<$ 0.001 ) and the degree of liver injury during treatment were independently associated with mortality in our patient cohort (group 1 vs. 2 , OR $=1.910,95 \%$ CI: $0.663-$ $5.498, p=0.231$; group 1 vs. $3, \mathrm{OR}=67.437,95 \% \mathrm{CI}$ : 8.432-539.341, $p<0.001$ ) (online suppl. Table; see www. karger.com/doi/10.1159/000510758 for all online suppl. material). Although ALF was associated with poor clinical outcome compared to the absence of liver injury, the same effect could not be observed in the case of mild liver injury.

\section{Aggravation of Liver Injury after Viral Clearance in a} Third of the Patients

Next, we focused on the temporal pattern of viral detection and liver injury. Data on viral detection in the respiratory tract in conjunction with peak liver injury were available in 52 cases, 46 in the second group and 6 in the third group. In a third of the patients with mild liver injury and in half of the ALF patients, peak liver injury was observed at a time point when the virus was no longer detectable in the respiratory tract. In Figure 1, we show the timeline of viral detection and liver injury in the ALF group. The duration of viral shedding was comparable between the 3 groups.

Liver biopsy was available in 2 patients. We could not detect SARS-CoV-2 RNA using PCR in any of the 2 biopsies although the virus was detectable in a nasopharyngeal swab and in stool in the first patient and in an endotracheal aspirate but not in the blood in the second patient. The first patient showed signs of liver and spleen infarction as well as pulmonary embolism in a computer tomography and developed ALF. The histopathological examination of the first liver biopsy showed extended hepatocyte necrosis $(80 \%)$ with chronic cholestasis signs 


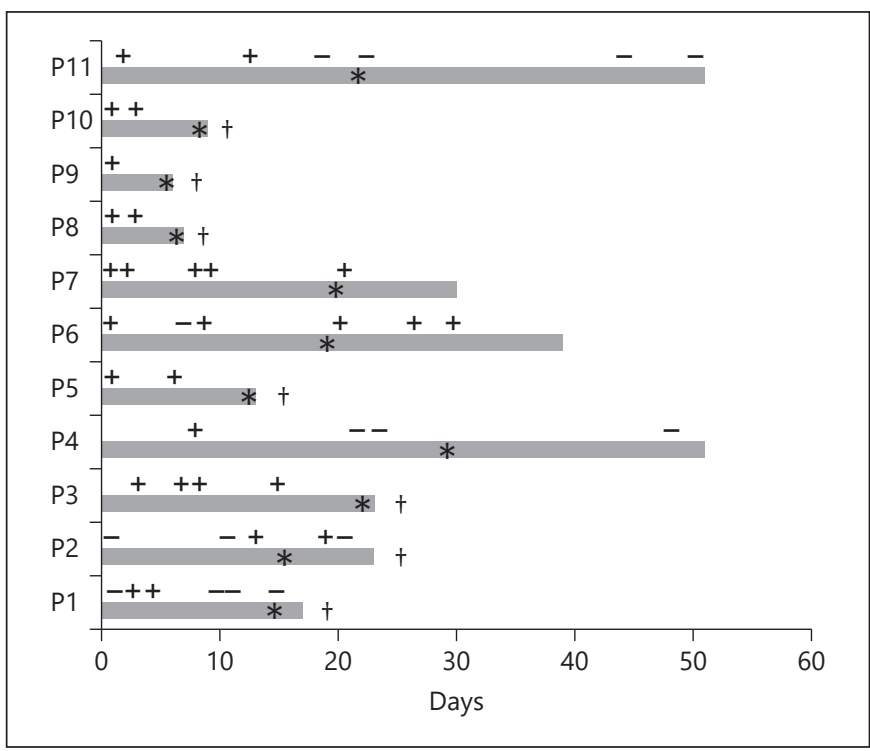

Fig. 1. Timeline of COVID-19 detection in the respiratory tract and peak liver injury in ALF patients. $\dagger$, death; ${ }^{*}$, peak liver injury; +, SARS-CoV-2 detected; -, SARS-CoV-2 undetectable.

without significant fibrosis or inflammation. The second patient did not meet the criteria of ALF, while the histopathological examination of the liver biopsy showed severe acute cholestasis signs combined with mild steatosis (5\% macrovesicular and 5\% microvesicular) and lymphocytes infiltration.

\section{Discussion}

Elevated ALT and/or bilirubin were observed in 63\% of our patients, in contrast to $13-41 \%$ reported in 4 large Chinese cohorts $[3,4,12,13]$. Abnormal liver parameters were even more frequently observed in our cohort, in 83 and $88 \%$ of the patients at admission and during treatment, respectively. The prevalence in our cohort was higher that what has been reported before in Chinese cohorts but also in a recent French study $[6,7,12]$.

Acute vascular events including mesenteric ischemia, myocardial infarction, pulmonary embolism, deep vein thrombosis, and stroke were common in our cohort $(24 / 147,16.3 \%)$ and especially in the ALF group (4, $36.4 \%)$. Although ALF patients were younger than patients without or with mild liver injury, they were more often diagnosed with acute vascular events, and the difference, however, did not reach statistical significance. All ALF patients presented with or developed sepsis and
MODS. Interestingly, ALF patients were exclusively male, indicating that male sex may be a risk factor for ALF in SARS-CoV-2 infection, contrary to what has been shown for ALF due to other pathologies $[14,15]$.

Unsurprisingly, ALF was associated with increased mortality. However, patients with mild liver injury during treatment had similar mortality rates with patients without liver injury, which suggests that the relationship between liver injury and mortality in COVID-19 patients is not binary. Rather, the extent of liver injury seems to be crucial for the outcome. The presence of any abnormal liver value at admission had no predictive value for mortality in our cohort, in contrast to a recent study from France [6]. It is, however, possible that our study had not enough power to detect this effect since only $17 \%$ of our patient presented with normal liver parameters on admission.

In $50 \%$ of ALF patients, the virus was no longer detectable in the respiratory tract at peak liver injury. A similar phenomenon was seen in the mild liver injury group with only $70 \%$ of the patients demonstrating detectable SARSCoV-2 RNA at peak liver injury. The aggravation of liver injury after viral clearance in a significant percentage of our cohort speaks against a predominant direct SARS$\mathrm{CoV}-2$ hepatopathic effect. While this cannot definitively exclude SARS-CoV-2 replication in the hepatocytes, the pattern of liver injury, our failure to detect viral RNA with a highly sensitive method (PCR) in the liver biopsies, the similar viral detection interval in all groups, and the previously reported absence of association of symptoms duration and liver injury [16] do not fit well with the hypothesis of direct viral contribution to liver impairment [17]. Indeed, severe liver injury in our cohort was seen as part of MODS with acute vascular events, possibly drugrelated and immune-mediated liver injury contributing to it. This is in accordance with previous observations stating that the cytokine storm observed in COVID-19 patients can lead to multiorgan failure, which includes the liver, but in general hepatic manifestations tend to be mild and transient [18]. On the other hand, in patients with pre-existing liver disease, SARS-CoV-2 infection is reported to have a worse outcome but not an increased incidence compared to patients without pre-existing liver disease $[19,20]$.

In conclusion, mild liver injury was not associated with worse outcome in our cohort, and the pattern of liver injury did not fit well to the theory of SARS-CoV-2 directly causing liver impairment. Instead, severe liver injury in our cohort was associated MODS and acute vascular events.
Anastasiou/Korth/Herbstreit/Witzke/ Lange 


\section{Statement of Ethics}

This retrospective study was carried out in accordance with the Declaration of Helsinki and the guidelines of the International Conference for Harmonization for Good Clinical Practice. It was approved by the local ethics committee at the University Hospital Essen (Institutional Review Board). Informed consent was waived due to the retrospective character of the study.

\section{Conflict of Interest Statement}

O.A. has received a research grant from Hexal, unrelated to the submitted work. J.K. has received grants, speaker's fees, honoraria, and travel expenses from Astellas, Basilea, Chiesi, Janssen, Novartis, and Roche. F.H. has nothing to disclose. O.W. has received research grants for clinical studies, speaker's fees, honoraria, and travel expenses from Amgen, Alexion, Astellas, Basilea, Biotest,
Bristol-Myers Squibb, Correvio, Chiesi, Gilead, Hexal, Janssen, Dr. F. Köhler Chemie, MSD, Novartis, Roche, Pfizer, Sanofi, TEVA, and UCB.C.M.L. has received speaker fees from AbbVie, Gilead, MSD, and Norgine and travel support from AbbVie and Gilead, all unrelated to the submitted work.

\section{Funding Sources}

O.W. is supported by an unrestricted grant of the RudolfAckermann-Stiftung (Stiftung für Klinische Infektiologie).

\section{Author Contributions}

O.E.A. designed the study, analyzed data, and wrote the manuscript. J.K., F.H., O.W., C.M.L. produced data and helped to draft the manuscript.

\section{References}

1 Stang A, Standl F, Jöckel K-H. Characteristics of COVID-19 pandemic and public health consequences. Herz. 2020;45(4):313-5.

2 Welfens PJJ. Macroeconomic and health care aspects of the coronavirus epidemic: EU, US and global perspectives. Int Econ Econ Policy. 2020:1-68.

3 Fan Z, Chen L, Li J, Cheng X, Yang J, Tian C, et al. Clinical features of COVID-19-related liver damage. Clin Gastroenterol Hepatol. 2020 Apr 10.

4 Guan WJ, Ni ZY, Hu Y, Liang WH, Ou CQ, $\mathrm{He}$ JX, et al. Clinical characteristics of coronavirus disease 2019 in China. N Engl J Med. 2020 Apr 30;382(18):1708-20.

5 Wang D, Hu B, Hu C, Zhu F, Liu X, Zhang J, et al. Clinical characteristics of 138 hospitalized patients with 2019 novel coronavirus-infected Pneumonia in Wuhan, China. JAMA. 2020;323(11):1061-9.

6 Meszaros M, Meunier L, Morquin D, Klouche K, Fesler P, Malezieux E, et al. Abnormal liver tests in patients hospitalized with coronavirus disease 2019: should we worry? Liver Int. 2020 Jun 3.

7 Xu X-W, Wu X-X, Jiang X-G, Xu K-J, Ying $\mathrm{L}-J, \mathrm{Ma} C-\mathrm{L}$, et al. Clinical findings in a group of patients infected with the 2019 novel coronavirus (SARS-Cov-2) outside of Wuhan, China: retrospective case series. BMJ. 2020; 368:m606.
8 Zhang Y, Zheng L, Liu L, Zhao M, Xiao J, Zhao Q. Liver impairment in COVID-19 patients: a retrospective analysis of 115 cases from a single centre in Wuhan city, China. Liver Int. 2020 Apr 2.

9 Cai Q, Huang D, Yu H, Zhu Z, Xia Z, Su Y, et al. COVID-19: abnormal liver function tests. J Hepatol. 2020 Apr 13.

10 Wendon J, Cordoba J, Wendon J, Larsen FS, Cordoba J, Dhawan A, et al. EASL clinical practical guidelines on the management of acute (fulminant) liver failure. J Hepatol. 2017;66(5):1047-81.

11 Giannini EG, Testa R, Savarino V. Liver enzyme alteration: a guide for clinicians. CMAJ. 2005;172(3):367-79.

12 Cai Q, Huang D, Yu H, Zhu Z, Xia Z, Su Y, et al. COVID-19: abnormal liver function tests. J Hepatol. 2020;73(3):566-74.

13 Wang Y, Liu S, Liu H, Li W, Lin F, Jiang L, et al. SARS-CoV-2 infection of the liver directly contributes to hepatic impairment in patients with COVID-19. J Hepatol. 2020 May 11.

14 Guy J, Peters MG. Liver disease in women: the influence of gender on epidemiology, natural history, and patient outcomes. Gastroenterol Hepatol. 2013;9(10):633-9.
15 Anastasiou OE, Widera M, Westhaus S, Timmer L, Korth J, Gerken G, et al. Clinical outcome and viral genome variability of hepatitis $B$ virus-induced acute liver failure. Hepatology. 2019 Mar 01;69(3):993-1003.

16 Shi H, Han X, Jiang N, Cao Y, Alwalid O, Gu $\mathrm{J}$, et al. Radiological findings from 81 patients with COVID-19 pneumonia in Wuhan, China: a descriptive study. Lancet Infect Dis. 2020 Apr 01;20(4):425-34.

17 Bangash MN, Patel J, Parekh D. COVID-19 and the liver: little cause for concern. Lancet Gastroenterology Hepatology. 2020;5(6): 529-30.

18 Pawlotsky J-M. COVID-19 and the liver-related deaths to come. Nat Rev Gastroenterology Hepatol. 2020 Jun 11:1-3.

19 Ji D, Zhang D, Yang T, Mu J, Zhao P, Xu J, et al. Effect of COVID-19 on patients with compensated chronic liver diseases. Hepatol Int. 2020 Jul 30:1-10.

20 Singh S, Khan A. Clinical characteristics and outcomes of coronavirus disease 2019 among patients with preexisting liver disease in the United States: a multicenter research network study. Gastroenterology. 2020 Aug; 159(2): 768-71.e3. 